\title{
Ovarian stimulation drugs alter the metabolite content of the growing follicle: in vivo spectroscopic evaluation of follicle fluid
}

\author{
(D) Nur Dokuzeylül Güngör1 ${ }^{1}$, (D Kağan Güngör² \\ ${ }^{1}$ Clinic of Reproductive Endocrinology and IVF, BAU Medical Park Göztepe Hospital, İstanbul, Turkey \\ 2Department of Endocrinology and Metabolism, İstanbul Medeniyet University, Göztepe Training and Research Hospital, \\ İstanbul, Turkey
}

\section{Abstract}

Objective: To determine and compare metabolite content using magnetic resonance spectroscopy (MRS) of growing follicles in patients with polycystic ovary syndrome (PCOS) receiving recombinant follicle stimulating hormone ( $\mathrm{rSSH}$ ), clomiphene citrate (CC) or aromatase inhibitor (AI) for ovarian stimulation.

Material and Methods: Thirty patients diagnosed with PCOS and infertility and scheduled for ovarian stimulation were divided by therapy, $\mathrm{rFSH} / \mathrm{CC} / \mathrm{AI}$, into three equal groups. Five fertile cases were designated as the control group. When the follicle diameters reached 16-18 mm in each group, patients underwent MRS and the metabolite content of a dominant follicle was analyzed. N-acetylaspartate, lactate (Lac), creatine $(\mathrm{Cr})$, and choline (Cho) metabolite levels in parts per million were measured in the spectra.

Results: A three-fold decrease in dominant follicle Cho content was found in patients receiving CC compared to control subjects. Similarly, the dominant follicle Cho intensities of patients given $\mathrm{rFSH}$ and $\mathrm{AI}$ were noted to be significantly higher than those who received CC. Only dominant follicle Lac levels of the patients who received CC were found to be significantly higher than the other groups. Cr peak intensities of patients receiving $\mathrm{CC}$ were found to be approximately three times less than control subjects. Cr signal intensity was significantly higher in patients receiving $\mathrm{rFSH}$ or $\mathrm{AI}$ than in patients receiving $\mathrm{CC}$. While two patients became pregnant in the CC group, three patients in the $\mathrm{AI}$ group and five patients in the rFSH group became pregnant. The main metabolites detected in patients who conceived in each group were Cho and Cr. In cases who could not conceive, while Lac and lipid signals increased, Cho and Cr signals decreased.

Conclusion: Unlike CC, ovarian stimulation with rFSH or AI does not alter dominant follicle metabolite content. The developmental capacity of a growing egg may be determined non-invasively with MRS. (J Turk Ger Gynecol Assoc 2021; 22: 132-8)

Keywords: MR spectroscopy, $\mathrm{rFSH}$, aromatase inhibitor, clomiphene citrate, dominant follicle

Received: 29 June, 2020 Accepted: 09 December, 2020

\section{Introduction}

Drugs such as recombinant follicle stimulating hormone (rFSH), clomiphene citrate (CC) and aromatase inhibitor (AI), which are used for ovarian stimulation, have all the features necessary for the selection, growth and numerical increase of eggs. However, sometimes these drugs may lead to the formation and selection of poor quality oocytes, resulting in poor quality embryos (1-4). Letrozole, an AI, has a reversible effect and can block estrogen synthesis from androgens (4). While rFSH directly promotes follicle development, $\mathrm{CC}$ and $\mathrm{AI}$ stimulate follicle growth indirectly by increasing endogenous FSH release. Both letrozole and $\mathrm{CC}$ provide adequate follicle development when used for five days between $3^{\text {rd }}$ and $7^{\text {th }}$ days of the cycle.

However, in addition to the number of retrieved oocytes, both the quality of oocytes and the pregnancy rates obtained 
with these drugs are different (1). The main reason for this difference between induction drugs may be changes in follicle metabolism and development depending on the drug dose and mechanism of action. Both the agonistic and antagonistic effects of $\mathrm{CC}$ on estrogen receptors may affect follicle developmental capacity independently of endogenous FSH levels. Likewise, the aromatase enzyme inhibitory effect of letrozole may disturb estrogen production capacity of granulosa cells that may, in turn, lead to unfavorable consequences on follicle developmental potential (1-4). In addition, use of these drugs may also affect the morphological and receptive properties of the endometrium $(1,2)$. However, there are not enough clinical data regarding the impact of these drugs on developing follicle metabolite composition. Clinicians therefore have to act on the basis of clinical and embryological data and experience rather than molecular data when deciding on which group of drug to use.

It is not possible to make a clear comment about follicle quality based on images obtained during folliculometry. For this reason, there is a need for a method to evaluate the developmental potential of the follicle non-invasively during ovarian stimulation cycles. A study by Wallace et al. (5) reported a positive correlation between follicle fluid metabolite content and oocyte developmental capacity. However, this study used invasive techniques because the follicle liquid was removed for in vitro analysis. Therefore, an oocyte pick-up (OPU) was required, which is an invasive procedure. In addition, since a nuclear magnetic resonance process was performed after OPU, there was no possibility of leaving the developing egg in vivo and undisturbed. To eliminate all these disadvantages and to evaluate egg development in vivo, the only non-invasive method available is magnetic resonance spectroscopy (MRS). It has been reported that MRS can detect metabolite content in biological fluids with great accuracy (6). MRS can analyze the metabolic changes in a living cell using conventional MR imaging. The primary advantage of this technique is its non-invasive nature and cost. MRS can measure various intrafollicular molecules that are present at low parts per million (ppm) levels. Although the method was originally developed for brain lesions, its use in reproductive organs, such as endometrium, myometrium, and ovary has been reported by many authors (5-8). This technique also allows comparison of follicular fluid metabolite levels in different treatment regimens in vivo and non-invasively. Although there are many studies investigating the metabolite concentrations in the reproductive organs (5-10), there are no published studies investigating the effects of ovarian stimulation drugs on follicular fluid metabolite compositon. Therefore, the primary outcome of the study was to investigate whether $\mathrm{rFSH}, \mathrm{AI}$, and $\mathrm{CC}$ have an effect on metabolite content of growing follicle in women with polycystic ovary syndrome (PCOS) undergoing ovarian stimulation. The secondary outcome was to compare the possible relationship between the metabolite intensities obtained from the dominant follicle and clinical pregnancy rate.

\section{Material and Methods}

The study was conducted in the BAU Göztepe Medicalpark Hospital, Clinic of In-vitro Fertilization. Thirty-five women (30 PCOS and five fertile controls) between the ages of 19 and 37 years with a body mass index (BMI) of 18-29 were enrolled the study. The PCOS group, with primary infertility and anovulation, was divided in three equal groups, depending on antagonist protocol with $\mathrm{rFSH}(\mathrm{n}=10)$, or PCOS women receiving $\mathrm{CC}(\mathrm{n}=10)$ or $\mathrm{PCOS}$ women receiving $\mathrm{AI}(\mathrm{n}=10)$. The controls had similar ages and BMIs and did not receive any drugs for ovarian stimulation. The women in control group had at least one or more children. They had no history of clinical or laboratory findings of PCOS. Women in each PCOS treatment group met the diagnostic criteria for PCOS when at least two of the following three features was present (11): 1) Amenorrhea or oligomenorrhea with chronic anovulation, 2) clinical and/or biochemical evidence of hyperandrogenism, and 3) ultrasonographic appearance of PCOS (2). Participants with a history of current or previous use of metformin, tubal or male factor infertility, evidence of any uterine, ovarian or peritoneal diseases, such as leiomyoma, benign ovarian cyst, endometrioma or endometriosis potentially affecting the ovarian microenvironment, were also excluded. This study has institutional review board approval.

PCOS participants on antagonist protocol were given daily rFSH starting from the third day of the menstrual cycle. Dose adjustments were performed according to BMI and ovarian response. The rFSH dose used ranged from 100 units to 187.5 units. Follicle development was monitored by using transvaginal ultrasonography and serum estradiol concentrations. As soon as one of the follicles reached $14 \mathrm{~mm}$ in diameter, gonadotropinreleasing hormone antagonist (cetrotide: $250 \mu \mathrm{g}$, Merck Serono, Turkey) was started and this treatment was continued until human chorionic gonadotropin (hCG) was administered. Oocyte maturation was triggered with recombinant hCG (ovitrelle, Merck-Serono, $250 \mathrm{mg}$, Modugno, Italy). As soon as the follicle diameters reached $16-18 \mathrm{~mm}$ in each group, patients underwent MRS and the metabolite content of a dominant follicle was analyzed. N-acetylaspartate (NAA), lactate (Lac), creatine ( $\mathrm{Cr}$ ), and choline (Cho) metabolite levels were measured in the spectra in ppm $(10,12)$.

PCOS subjects in the CC group were treated with CC (serophene, Serono, Rome, Italy) at dosage of $150 \mathrm{mg} /$ day for five days beginning from the third day of spontaneous or 
progesterone-induced withdrawal bleeding. Patients in the CC group were selected from CC-resistant PCOS patients. CC resistance was defined as failure to ovulate after six months of treatment at an appropriate dose. In our study, patients who could not achieve ovulation despite using $150 \mathrm{mg} /$ day clomiphene were considered CC resistant. PCOS subjects on AI group were treated with letrozole (Femara, Novartis, İstanbul, Turkey) at dosage of $2.5 \mathrm{mg} /$ day for five days beginning from the third day of a spontaneous or progesterone-induced menstrual bleeding. Follicle development was monitored using transvaginal ultrasound (TV-USG). Women on CC or letrozole also underwent spetroscopic analysis of one dominant follicle as soon as the detection of a follicle with a mean diameter of at least $16-18 \mathrm{~mm}$ on TV-USG examination. Women in the $\mathrm{CC}$ or letrozole group underwent either timed intercourse or intrauterine insemination. Fertile women on their natural cycle underwent spectroscopy when the dominant follicle had a mean diameter of at least $16-18 \mathrm{~mm}$.

\section{Spectroscopic analysis of dominant follicle}

Axial and coronal plane T1-weighted images of the dominant follicle with $5 \mathrm{~mm}$ thick sections were achieved using $1.5 \mathrm{~T}$ MRI. A single voxel MR spectroscopy procedure was applied with a short and long TE. As signals from other follicles may obscure the metabolite content of the dominant follicle, the selected voxel should be placed in an appropriate area so that it is possible to get clear information about the content of follicle fluid. Therefore, great care was taken to exclude neighboring follicles in the area of the selected voxel. Finally, the voxel was placed just in the middle of the dominant follicle, after ensuring no interference from neighbouring follicles or structures
The metabolite ratios of acquired signals from the dominant follicle were determined using MR User Interface software Automated Quantitation of Short Echo Time MRS spectra (13). NAA, Lac, Cr, and Cho peaks of each group were analysed. A detailed discussion of these spectroscopic methods can be found elsewhere $(6-9,12)$. The primary outcome of this study was to investigate the effects of $\mathrm{rFSH}, \mathrm{CC}$ and $\mathrm{AI}$ treatment on dominant follicle metabolite levels, in terms of NAA, $\mathrm{Lac}, \mathrm{Cr}$ and Cho. The secondary outcome was to compare the possible relationship between the metabolite intensities obtained from the dominant follicle and clinical pregnancy rate. All metabolite peaks were analyzed quantitatively and compared with results by either the timed intercourse, intrauterine insemination or embryo transfer methods. Clinical pregnancy rate was defined as evidence of a gestational sac confirmed by ultrasound examination.

\section{Statistical analysis}

Analyses of all data were performed on Statistical Package for Social Sciences (IBM Inc., Chicago, IL, USA). Q-Q and histogram plots or Kolmogorov-Smirnov test were used to determine whether variables were normally distributed. Data are given as mean \pm standard deviation or median for continuous variables according to normality of distribution. Normally distributed variables were analyzed with the Independent samples t-test. Non-normally distributed variables were analyzed with the Mann-Whitney U test. Categorical variables were analyzed with chi-square tests. A p $<0.05$ was considered to be significant.

\section{Results}

The clinical and demographic characteristics of the four groups were similar (Table 1). Three participants were excluded from

Table 1. Demographic characteristics and metabolites ratios of treatment and control groups

\begin{tabular}{|c|c|c|c|c|}
\hline & rFSH & Clomiphene & Letrozole & Fertile control \\
\hline Mean age (yrs) & $29.1 \pm 6.31$ & $28.3 \pm 1.40$ & $28.6 \pm 0.21$ & $29.2 \pm 2.45$ \\
\hline BMI $\left(\mathrm{kg} / \mathrm{m}^{2}\right)$ & $27.4 \pm 5.14$ & $26.5 \pm 5.32$ & $27.1 \pm 2.11$ & $27.5 \pm 3.30$ \\
\hline Etiology of infertility & PCOS & PCOS & PCOS & NA \\
\hline Mean follicle size (mm) & $18.4 \pm 0.3$ & $17.4 \pm 0.3$ & $18.2 \pm 2.6$ & $16.8 \pm 1.2$ \\
\hline \multicolumn{5}{|c|}{ Dominant follicle metabolite composition } \\
\hline 1. NAA & $1.18(0.9)$ & $1.32(1.1)$ & $1.40(0.8)$ & $1.10(0.7)$ \\
\hline 2. Lactate & $0.98(0.80)$ & $1.89(1.2)^{*}$ & $0.98(0.7)$ & $0.89(0.6)$ \\
\hline 3. Choline & $1.94(1.0)$ & $0.61(0.5)^{*}$ & $1.93(1.2)$ & $2.12(1.3)$ \\
\hline 4. Creatine & $1.91(0.9)$ & $0.84(0.7)^{*}$ & $1.92(1.2)$ & $2.33(1.3)$ \\
\hline
\end{tabular}


the study due to inadequate follicle growth in one fertile control and bad spectral image in two women receiving letrozole. The remaining 32 subjects in control and treatment groups had good spectral images. Compared to the fertile group, a significantly decreased Cho signal was found in the CC group. An approximately three-fold decline in Cho peak intensity was found in PCOS subjects compared to the Cho peak intensity of control participants [0.61 (0.5) ppm vs. 2.12 (1.3) ppm, respectively; $\mathrm{p}<0.01$ ]. Likewise, the Cho peak intensity in the $\mathrm{rFSH}$ and $\mathrm{AI}$ groups were higher than those in the CC group. The Cho peak intensity of fertile controls and patients receiving rFSH or letrozole were similar.

The Lac signal intensity of patients receiving $\mathrm{CC}$ was significantly higher than that of fertile controls [1.89 (1.2) vs. $0.89(0.6), \mathrm{p}<0.02]$ or patients receiving $\mathrm{rFSH}[0.98(0.80)$ vs. 1.89 (1.2), $\mathrm{p}<0.03$ ] or letrozole [0.98 (0.7) vs. 1.89 (1.2) $\mathrm{p}<0.01]$. An almost two-fold increase in Lac signal was detected after $\mathrm{CC}$ treatment compared to $\mathrm{rFSH}$, letrozole or fertile groups. In contrast, Lac signal intensities of fertile controls, and the rFSH and letrozole groups were similar. Follicle fluid $\mathrm{Cr}$ signal intensity of fertile group was approximately three times higher than $\mathrm{Cr}$ signal of patients receiving CC [2.33 (1.3) vs. 0.84 (0.7) $\mathrm{p}<0.01$ ]. Likewise, $\mathrm{Cr}$ signal intensity of women receiving $\mathrm{rFSH}$ or letrozole was significantly higher than those receiving $\mathrm{CC}$ ( $p<0.01$ and $p<0.01$, respectively). No significant difference was found between the fertile control, $\mathrm{rFSH}$, and letrozole groups in terms of $\mathrm{Cr}$ metabolite. The NAA content of fertile and treatment groups were found to be similar (Table 1 and Figure 1).

When a comparison was made between follicular fluid metabolite signal intensities and pregnancy rates, the following results were obtained. While clinical pregnancy was detected in 2 of 10 patients in the $\mathrm{CC}$ group, pregnancy could not be achieved in the remaining eight cases. While the most prominent metabolites were Cho and $\mathrm{Cr}$ in the two cases who became pregnant, in those who could not conceive, the Lac peak was most prominent. While pregnancy was achieved in 3 of 10 cases in the AI group, pregnancy was not detected in seven cases. While high Cho signal was detected in pregnant patients, weak Cho signal and high lipid signal were detected in those who could not conceive. While 5 out of 10 patients in the $\mathrm{FSH}$ group had clinical pregnancy, the remaining five cases did not become pregnant. High Cho and low Lac signal were typical findings in patients who became pregnant in this group. Weak Cho and Cr signal was striking in patients who could not conceive.
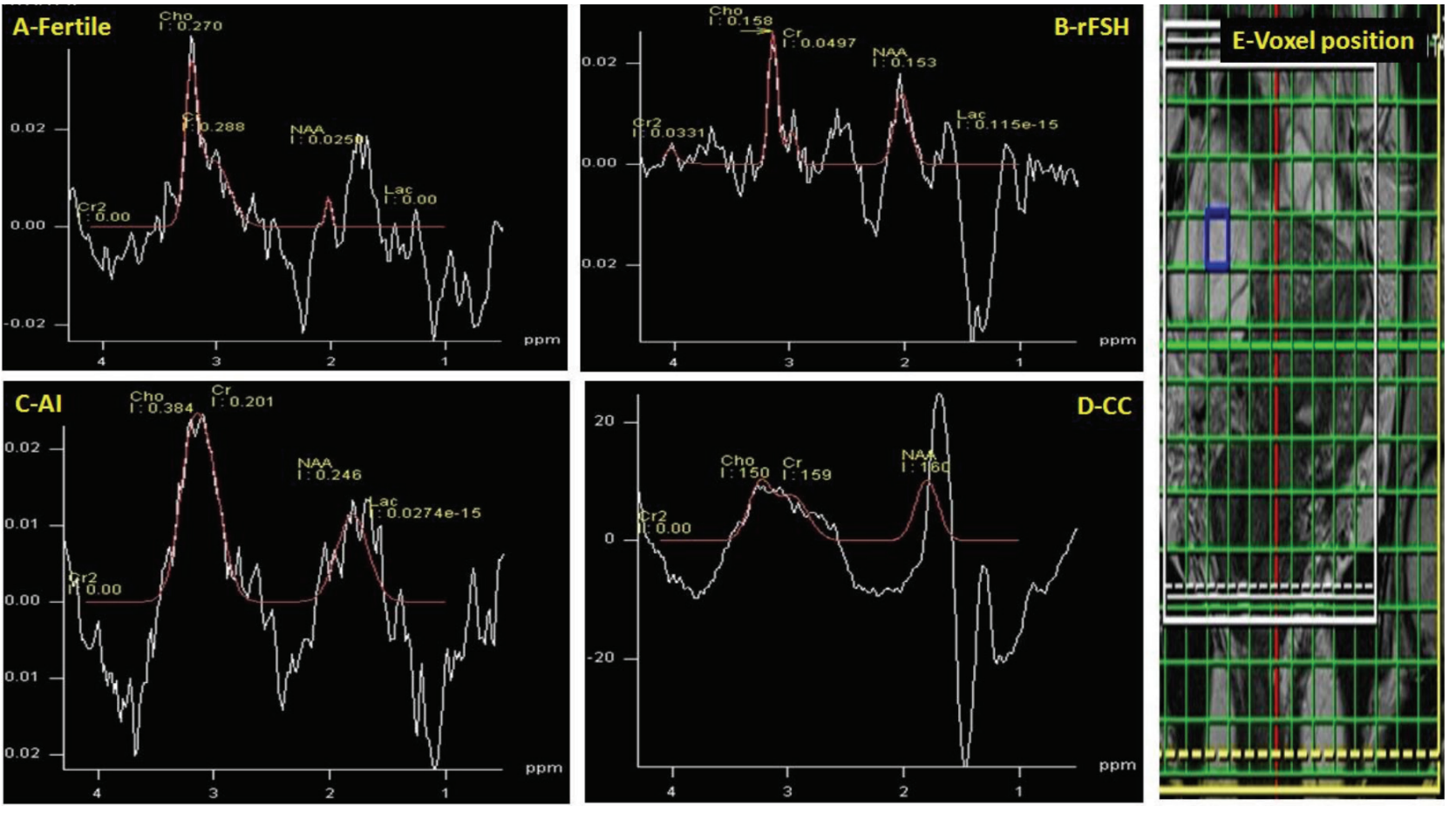

Figure 1.Spectroscopy analysis of fertile group (A) and participants receiving rFSH (B), aromatase inhibitor (C) or clomiphene citrate (D). An approximately four-fold decrease in Cho signal was detected in PCOS group receiving CC (D). Cho signal of subjects taking rFSH or AI were significantly higher than in the CC group. Signals from ovary and other pelvic structures may obscure the metabolite resonance of dominant follicle. Hence, the voxel was placed at the center of dominant follicle to avoid any signal contamination from neighboring tissues (E)

rFSH: Recombinant follicle stimulating hormone, NAA: N-acetylaspartate, Lac: Lactate, Cr: Creatine, Cho: Choline, AI: Aromatase inhibitor, CC: Clomiphene citrate, PCOS: Polycystic ovary syndrome 


\section{Discussion}

If a technique makes it possible to determine the best quality oocyte before OPU, pregnancy rates could be improved. Although morphological analysis of the oocyte is the basic method for determining embryo quality, it does not always correctly detect reproductive outcome $(14,15)$. Metabolite composition of developing follicle is an indicator of whether an embryo will complete its developmental process and reach the cleavage stage or not $(16,17)$. Although drugs used for ovarian stimulation improve maturation of oocytes, adverse effects of these drugs on natural selection steps can cause retrieval of developmentally incompetent oocytes. In good agreement with this, it has been reported that few of the embryos collected by OPU are competent to complete their developmental stages in a healthy manner and gain implantation potential (17). Fortunately, there is a new and non-invasive method to test the effects of ovarian stimulation drugs on the metabolite profile of the developing follicle, MRS. MRS is an advanced technology that enables the determination of metabolite content in all biological fluid layers in detail. This non-invasive method has been used in the evaluation of reproductive tissues including ovary and endometrium (6).

Follicle fluid has a nutritive effect on the growing follicle (18) so determining the metabolites in this fluid non-invasively would provide preliminary information about the metabolite content of the developing follicle. Studies have demonstrated that measurement of Cho and Lac metabolites in follicle fluid with nuclear MRS correlates with oocyte developmental capacity $(5,16)$. The metabolite composition of the cumulus oocyte complex in in vitro maturation cycles was described by Uhde et al. (19). After 23 hours of follow-up, 369 different metabolites were isolated in maturation medium. The metabolites which exhibited a significant increase in concentration during follicle growth include amino acids, saccharides, lipids, UDP-glucose, Lac, pyruvate and sphingomyelin. The two metabolites with the most significant increase in concentration were $\mathrm{Cr}$ and Lac. During 23 hours of maturation period, a 516-fold increase in $\mathrm{Cr}$ and a 1227-fold increase in Lac were reported. Lipids have a crtical role in energy production during oocyte maturation and the establishment of a developmentally mature oocyte (19). The transport of CC-derived pyruvate with lipids into the oocyte is critical for ATP production by the oocyte (20). Uhde et al. (19) showed that during oocyte maturation there was a significant increase in concentrations of both pyruvate and Lac in the medium, while glucose concentrations decreased. One possible reason for this decrease in glucose levels in follicle fluid would be increased glycolysis in cumulus cells during the maturation period.
In the current study, the effects of $\mathrm{rSSH}, \mathrm{CC}$, and $\mathrm{AI}$ on dominant follicle metabolite content were investigated for the first time. The main result was that the metabolite signals of growing follicle were quite different, depending on which treatment had been given, even though the follicle size was sufficient on ultrasonographic examination. The metabolite composition of dominant follicles of women receiving $\mathrm{AI}$ or $\mathrm{rSSH}$ were not different from those of fertile patients. On the other hand, the metabolite signals of patient receiving $\mathrm{CC}$ were different from that of fertile control, $\mathrm{rFSH}$ or AI groups. The $\mathrm{Cho}, \mathrm{Cr}$, and $\mathrm{Lac}$ signals obtained from subjects receiving $\mathrm{CC}$ were significantly different from the signals of $\mathrm{rFSH}$ and $\mathrm{AI}$ groups. Of the four measured metabolites, only NAA signals were similar between the groups. Additionally, the intensity of the Lac signal of the dominant follicle was elevated in the CC group compared with $\mathrm{rFSH}$ or $\mathrm{AI}$ group. Administarion of $\mathrm{CC}$ for ovarian stimulation disturbed the release of $\mathrm{Cho}$ and $\mathrm{Cr}$ that are basic metabolites produced by the developing follicle. The mechanism of impaired metabolite signals in patients using $\mathrm{CC}$ is not clear. This adverse effect may be due to the estrogen receptor blocking effect of $\mathrm{CC}$. CC has been shown to have a negative impact on the endometrium (1). Despite high ovulation rates, low clinical pregnancy rates in women given $\mathrm{CC}$ may be due to failed follicle development. Both $\mathrm{AI}$ and $\mathrm{CC}$ stimulate follicle development by increasing endogenous FSH levels. However, AI reduces estrogen levels by blocking the aromatase enzyme and leads to an increase in FSH levels. CC increases FSH levels by binding to estrogen receptors. Since CC binds to all estrogen receptors, it also binds to estrogen receptors in cumulus cells. Thus, while CC contributes to follicle development via FSH, it also negatively affects follicle development by blocking estrogen effects in the cumulus cells. Since AI does not block estrogen receptors, it is unsurprising that different follicular growth patterns are seen in women treated with $\mathrm{AI}$ and with CC.

The increase in follicular fluid Lac levels may have both negative and positive effects on follicle development. High levels of follicle fluid Lac may lead to anaerobic glycolysis and impair healthy oocyte development in women on CC (68). Interestingly, exposure of developing mouse oocytes to a high fat diet results in failed oocyte nuclear maturation (21). Likewise, decreased levels of Cho metabolite in women on CC can be a sign of defective follicle development. $\mathrm{Cr}$ signal is an important indicator of energy metabolism of living cells (6-8). Low $\mathrm{Cr}$ signals in women receiving $\mathrm{CC}$ may be another indicator of impaired follicle maturation and can be associated with poor follicle morphology. In contrast, the increase in follicular fluid Lac levels in the CC group should not be interpreted as an absolute pathological state. Increase in follicular fluid Lac levels is also detected in natural follicle development 
processes. Especially in in vitro maturation cycles, during the first eight hours of follow-up, a very significant increase in Lac concentration is detected in the maturation medium. It has been reported that the Lac concentration in the maturation medium increased 667-fold during in vitro maturation (19). What is important here is whether the oocyte is able to enzymatically transport Lac rather than the increase in fluid Lac levels, per se. Hérubel et al. (22) showed that oocytes highly express monocarboxylic acid transporters that enable the transport of Lac between cumulus cells and the oocyte. If the increase in Lac levels during follicle development exceeds the transport capacity of the available monocarboxylic acid transporters, the rate of cell growth and proliferation will slow down. Since the high Lac increase seen in the CC group is above the carrying capacity of the follicle, the cumulus cells will try to compensate for the Lac increase by slowing cell proliferation (7). Since Cho is a marker of cell membrane turnover, the amount of Cho passing into follicular fluid will also decrease due to decreased cumulus cell proliferation.

\section{Conclusion}

It is possible to predict the developmental capacity of an egg before OPU by determining metabolite content of a growing follicle specifically and accurately with spectroscopy. This is important as it would aid in selection of the best quality embryo and would aid in overcoming immature and incompetent oocyte selection. Thus, the selection of oocytes that have completed their developmental process before embryo transfer is possible and the transfer decision can be made accordingly.

Ethics Committee Approval: Local ethical committee report was received from the BAU Göztepe Medicalpark Hospital Medical Director.

Informed Consent: Verbal and written informed consent was obtained.

Peer-review: Externally peer-reviewed.

Author Contributions: Surgical and Medical Practices: N.D.G.; Concept: N.D.G., K.G.; Design: N.D.G., K.G.; Data Collection or Processing: N.D.G., K.G.; Analysis or Interpretation: N.D.G., K.G.; Literature Search: N.D.G., K.G.; Writing: N.D.G.

Conflict of Interest: No conflict of interest is declared by the authors.

Financial Disclosure: The authors declared that this study received no financial support.

\section{References}

1. Haas J, Casper RF. In vitro fertilization treatments with the use of clomiphene citrate or letrozole. Fertil Steril 2017; 108: 568-71.

2. Adashi EY. Clomiphene citrate: mechanism(s) and site(s) of action-a hypothesis revisited. Fertil Steril 1984; 42: 331-44.

3. Casper RF. Aromatase inhibitors in ovarian stimulation. J Steroid Biochem Mol Biol 2007; 106: 71-5.

4. Casper RF, Mitwally MF. A historical perspective of aromatase inhibitors for ovulation induction. Fertil Steril 2012; 98: 1352-5.

5. Wallace M, Cottell E, Gibney MJ, McAuliffe FM, Wingfield M, Brennan L. An investigation into the relationship between the metabolic profile of follicular fluid, oocyte developmental potential, and implantation outcome. Fertil Steril 2012; 97: 1078-84.

6. Celik O, Hascalik S, Sarac K, Meydanli MM, Alkan A, Mizrak B. Magnetic resonance spectroscopy of premalignant and malignant endometrial disorders: a feasibility of in vivo study. Eur J Obstet Gynecol Reprod Biol 2005; 118: 241-5.

7. Hascalik S, Celik O, Sarac K, Meydanli MM, Alkan A, Mizrak B. Metabolic changes in pelvic lesions: findings at proton MR spectroscopic imaging. Gynecol Obstet Invest 2005; 60: 121-7.

8. Sarac K, Celik O, Hascalik S, Alkan A, Mizrak B. In vivo proton magnetic resonance spectroscopy in the evaluation of the endometrium. Acta Obstet Gynecol Scand 2004; 83: 751-7.

9. Ersahin A, Celik O, Acet M, Ersahin S, Acet T, Bozkurt DK, et al. Impact of Endometrioma Resection on Eutopic Endometrium Metabolite Contents: Noninvasive Evaluation of Endometrium Receptivity. Reprod Sci 2017; 24: 790-5.

10. Acet M, Celik N, Acet T, Ilhan S, Yardim M, Aktun HL, et al. Serum and follicular fluid irisin levels in poor and high responder women undergoing IVF/ICSI. Eur Rev Med Pharmacol Sci 2016; 20: 1940-6.

11. Rotterdam ESHRE/ASRM-sponsored PCOS consensus workshop group. Revised 2003 consensus on diagnostic criteria and longterm health risks related to polycystic ovary syndrome. Fertil Steril 2004; 81: 19-25.

12. Çelik Ö, Hatırnaz Ş, Erşahin A, Başbuğ A, Yetkin Yıldırım G, Özener $\mathrm{V}$, et al. Testis spectroscopy may predict sperm retrieval rate in men with non-obstructive azoospermia undergoing micro-TESE: A pilot study. J Turk Ger Gynecol Assoc 2020; 21: 70-8.

13. Poullet JB, Sima DM, Simonetti AW, Se Neuter B, Vanhamme L, Lemmerling $\mathrm{P}$, et al. An automated quantitation of short echo time MRS spectra in an open source software environment: AQSES. NMR Biomed 2007; 20: 493-504.

14. Sinclair KD, Lea RG, Rees WD, Young LE. The developmental origins of health and disease: current theories and epigenetic mechanisms. Soc Reprod Fertil Suppl 2007; 64: 425-43.

15. Singh R, Sinclair KD. Metabolomics: approaches to assessing oocyte and embryo quality. Theriogenology 2007; 68(Suppl 1): S5662.

16. Piñero-Sagredo E, Nunes S, de los Santos MJ, Celda B, Esteve V. NMR metabolic profile of human follicular fluid. NMR Biomed 2010; 23: 485-95

17. Kovalevsky G, Patrizio P. High rates of embryo wastage with use of assisted reproductive technology: a look at the trends between 1995 and 2001 in the United States. Fertil Steril 2005; 84: 325-30.

18. Edwards RG. Follicular fluid. J Reprod Fert 1974; 37: 189-219.

19. Uhde K, van Tol HTA, Stout TAE, Roelen BAJ: Metabolomic profiles of bovine cumulus cells and cumulus-oocyte-complexconditioned medium during maturation in vitro. Sci Rep 2018; 8 : 9477.

20. Simerman AA, Hill DL, Grogan TR, Elashoff D, Clarke NJ, Goldstein $\mathrm{EH}$, et al. Intrafollicular cortisol levels inversely correlate with cumulus cell lipid content as a possible energy source during oocyte 
meiotic resumption in women undergoing ovarian stimulation for in vitro fertilization. Fertil Steril 2015; 103: 249-57.

21. Yang X, Wu LL, Chura LR, Liang X, Lane M, Norman RJ, et al. Exposure to lipid-rich follicular fluid is associated with endoplasmic reticulum stress and impaired oocyte maturation in cumulusoocyte complexes. Fertil Steril 2012; 97: 1438-43.
22. Hérubel F, El Mouatassim S, Guérın P, Frydman R, Ménézo Y. Genetic expression of monocarboxylate transporters during human and murine oocyte maturation and early embryonic development. Zygote 2002; 10: 175-81. 